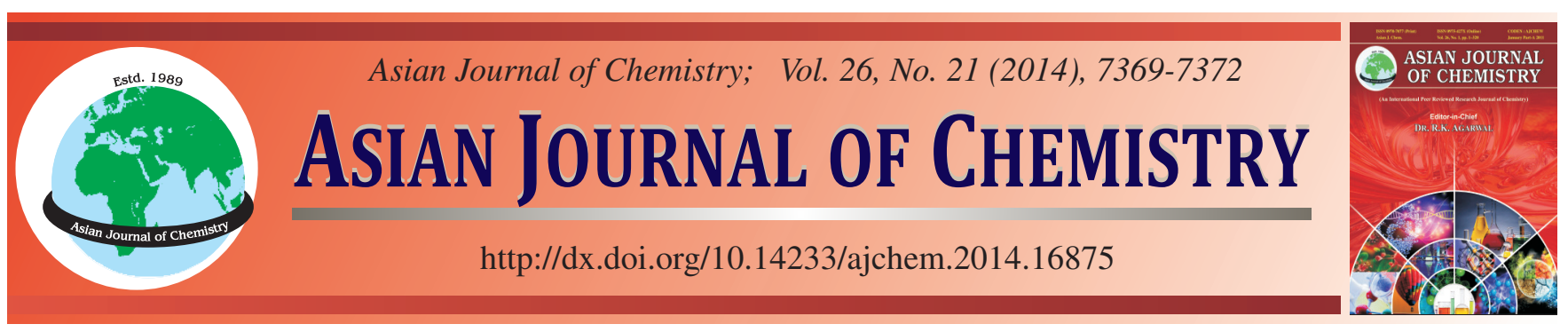

\title{
Preparation and Characterization of Blood Substitutes Based on Amphiphilic Biodegradable Copolymers
}

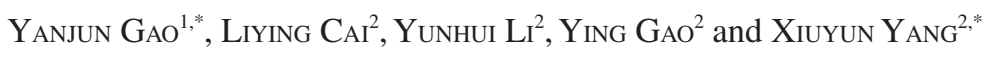

${ }^{1}$ Institute of Electronic Science and Technology, Jilin University, Changchun, P.R. China

${ }^{2}$ School of Chemistry and Environmental Engineering, Changchun University of Science and Technology, Changchun, P.R. China

*Corresponding authors: Tel/Fax: +86 431 85168382,+86 431 85582361; E-mail: 1370438405@qq.com; yangxiuyun@cust.edu.cn

\begin{abstract}
A biodegradable hemoglobin nanoparticles based on the copolymers of MPEG- $b$-PLA was prepared by self-assemble. The optimization conditions were obtained that the hemoglobin was encapsulated in the polymersomes. The hemoglobin nanoparticles with different states encapsulated in the polymersomes can be mutual transformed under different gas atmosphere was observed by UV spectra. While the oxygen partial pressure was increased, the oxy-hemoglobin was gradually transformed from deoxy-hemoglobin.
\end{abstract}

Keywords: Biodegradable copolymers, Polymersomes, Hemoglobin, Blood substitutes.

\section{INTRODUCTION}

Clinical blood transfusion has become a kind of general and secure and routine medical treatment method since Landsteiner discovered the human erythrocyte blood types in 1901. However, there is risks in the blood transfusion process. During clinical blood transfusion, there is various kinds of given or latent trouble and defect ${ }^{1,2}$. Therefore, blood substitutes studies have significant practical value to blood source shortage and transfusion of the infectious diseases.

Although blood substitutes with all the blood functions still can't be manufactured, substitutes with part functions have already developed, such as blood substitutes, erythrocyte substitutes etc. Blood substitutes actually mean erythrocyte substitutes with carrying oxygen capacity generally, which also called man-made oxygen carrier. Sellards had made the experiments of injecting soluble erythrocyte to human body in $1916^{3-5}$. Hence some scientists proposed that erythrocyte membrane was the virulence factors which could make human body to failure of the kidney function after injecting hemolysis substance ${ }^{6}$. New biodegradable hemoglobin can be fabricated by using the biodegradable block polymer material as host material combine with self-assembly technique which has lots of advantages such as adjustable size, mechanical capacity better than liposome, biodegradable ability and little hemoglobin leakage.

\section{EXPERIMENTAL}

Tin(II) 2-ethylhexanoate [ $\mathrm{Sn}(\mathrm{Oct})_{2}, 90 \%$ in 2-ethylhexanoic acid) from Strem Chemicals. Rhodamine B, sodium ascorbate and sodium dithionite were from Aladdin Co. Ltd. Bovine hemoglobin (Mw 64 500) was purchased from Shanghai Kayon Biological Technology Co. Ltd. and stabilized under $\mathrm{CO}$ atmosphere to afford carbonylated hemoglobin (COHb). Carbon monooxide (99.95\%) was from Dalian Date Gas Co. Ltd. Toluene was purified by distillation from sodium with benzophenone. Other solvents were analytical grade and used as received.

${ }^{1} \mathrm{H}$ NMR spectra were recorded with a Bruker AV400 spectrometer at $25^{\circ} \mathrm{C}$. Gel permeation chromatography (GPC) measurements were conducted with a Waters 410 GPC instrument equipped with a Waters Styragel HT6E column and a differential refractometer detector. DMF was used as eluent at a flow rate of $1 \mathrm{~mL} \mathrm{~min}^{-1}$ at $35^{\circ} \mathrm{C}$. Transmission electron microscopy (TEM) measurements were performed on a JEOL JEM1011 electron microscope operating at an acceleration voltage of $100 \mathrm{KV}$. Dynamic light scattering (DLS) experiments were carried out with a DAMN EOS instrument equipped with a He-He laser at a scattering angle of $90^{\circ}$. UV spectra were recorded on a UV-visible spectrophotometer (Shimadzu UV2450) at room temperature.

Synthesis of amphipathy diblock copolymer PEG- $b$ PLA: The block copolymer MPEG- $b$-PLA was synthesized by ring-oping polymerization of lactic acid in the presence of MPEG-OH as macroinitiator with $\mathrm{Sn}(\mathrm{Oct})_{2}$ as the catalyst at $110{ }^{\circ} \mathrm{C}$ for $10 \mathrm{~h}$ in toluene. The following is a typical example of synthesis of MPEG(1 K)-b-PLA(5 K). After a certain amount of MPEG (1 g, $1 \mathrm{mmol})$ was dried by toluene azeotropic distillation for $1 \mathrm{~h}$, lactic acid ( $5 \mathrm{~g}, 34.7 \mathrm{mmol})$ was added into the 
above system, followed by argon-purging three times. After sealing the system, prescribed amount of $\mathrm{Sn}(\mathrm{Oct})_{2}(0.5 \mathrm{~mol} \%$ of the total monomers) was added using a glass syringe. The reaction mixture was then heated to $120^{\circ} \mathrm{C}$ and stirred at this temperature for $12 \mathrm{~h}$. purification was performed by precipitating the reaction mixture against large excess of diethyl ether. The block copolymer was collected and dried in vacuum for $8 \mathrm{~h}$.

Preparation of micelles: The polymeric micelles were prepared using a dialysis method. Firstly, $5 \mathrm{mg}$ of graft copolymer PEG- $b$-PLA was dissolved in $10 \mathrm{~mL}$ of THF. Then the polymer solution was added to $20 \mathrm{~mL}$ of distilled water at a rate of $0.3 \mathrm{~mL} \mathrm{~min}^{-1}$. After being stirred at room temperature for $4 \mathrm{~h}$, THF was removed by dialysis against distilled water for 3 days using a dialysis bag (MWCO $3500 \mathrm{Da}$ ).

Preparation of polymersome-encapsulated hemoglobin: The polymersome-encapsulated hemoglobin was prepared according to the method described by Agashe et al. with some modifications ${ }^{7,8}$. First, the polymersome was obtained using the dialysis method described before. Then it was subjected to lyophilization for $48 \mathrm{~h}$ and stored at $-20{ }^{\circ} \mathrm{C}$ before use. To encapsulate hemoglobin into the polymersome, a typical procedure was described as follows. $10 \mathrm{mg}$ of dried polymersome was added into a vial which had been purged with $\mathrm{CO}$ for $0.5 \mathrm{~h}$, then $20 \mathrm{~mL}$ of $\mathrm{COHb}$ solution $(0.9 \%$ saline $)$ was added using a syringe. The solution was stirred for another $24 \mathrm{~h}$. Free hemoglobin was removed via diafiltration through a $500 \mathrm{kDa}$ hollow fiber (HF) membrane. The filtrate was assayed for the absence of hemoglobin via UV-visible spectroscopy. To the retentate was added $5 \mathrm{mg}$ of sodium ascorbate and it was then pluged $\mathrm{CO}$ for $1 \mathrm{~h}$. Finally the vial was sealed and stored at $4{ }^{\circ} \mathrm{C}$ for future use.

Hemoglobin encapsulation efficiency: The hemoglobin encapsulation efficiency was measured indirectly using the centrifugation method (15 $000 \mathrm{rpm} \times 3 \mathrm{~min})$ and the hemoglobin concentration was assayed via UV-visible spectroscopy. The encapsulation efficiency of hemoglobin was calculated by the following equation ${ }^{9,10}$.

Encapsulation efficiency $(\%)=\left(\mathrm{Hb}_{\text {total }}-\mathrm{Hb}_{\text {free }}\right) / \mathrm{Hb}_{\text {total }} \times 100$

Gas-bingding capacity: The gas binding and release ability of hemoglobin at different atmosphere could be monitored via UV-visible spectrophotometry. The CO-stabilized hemoglobin $(\mathrm{CO}-\mathrm{Hb})$ were converted to oxy-hemoglobin by exposing the solution to visible light under $\mathrm{O}_{2}$ atmosphere, while the deoxygenated hemoglobin (deoxy-Hb) state was obtained by exposing the solution to visible light under $\mathrm{N}_{2}$ atmosphere with the addition of a trace amount of $\mathrm{Na}_{2} \mathrm{~S}_{2} \mathrm{O}_{4}$.

\section{RESULTS AND DISCUSSION}

Synthesis of MPEG-PLA: The amphiphilic biodegradable copolymers with the hydrophobic poly(lactic acid) and the hydrophilic MPEG were obtained using the solution polymerization. The opening-ring polymerization of lactic acid was initiated by MPEG so as to fabricate MPEG-PLA block copolymer which was analyzed by ${ }^{1} \mathrm{H} \mathrm{NMR}$ in $\mathrm{CDCl}_{3}$. The typical ${ }^{1} \mathrm{H}$ NMR was shown in Fig. 1. The $-\mathrm{CH}_{2}-\mathrm{CH}_{2}$ - hydrogen positions of MPEG chain were in the $3.6 \mathrm{ppm}$, whereas the $\mathrm{O}-\mathrm{CH}$ $\left(\mathrm{CH}_{3}\right)$ - two kinds of hydrogen positions of lactic acid were separately in $5.1 \mathrm{ppm}$ and $1.6 \mathrm{ppm}$. The molecular weight of poly(lactic acid) was calculated by the relative proportions of these characteristic peak integral area. The amphiphilic block copolymers between hydrophilic parts and hydrophilic parts with the different proportions were prepared by $\mathrm{MPEG}_{1 \mathrm{~K}}$ and $\mathrm{MPEG}_{2 \mathrm{~K}}$.

A typical gel permeation chromatogram of the copolymer $\mathrm{MPEG}_{1 \mathrm{k}}-\mathrm{PLA}_{5 \mathrm{k}}$ and $\mathrm{MPEG}_{2 \mathrm{k}}-\mathrm{PLA}_{10 \mathrm{k}}$ was shown in Fig. 2. The dispersion values obtained from GPC measurement were low for all copolymers, indicating that the copolymers did not have a broad molecular weight distribution.<smiles>COC(C)C(=O)C(C)OCCC(C)(C)OC</smiles>

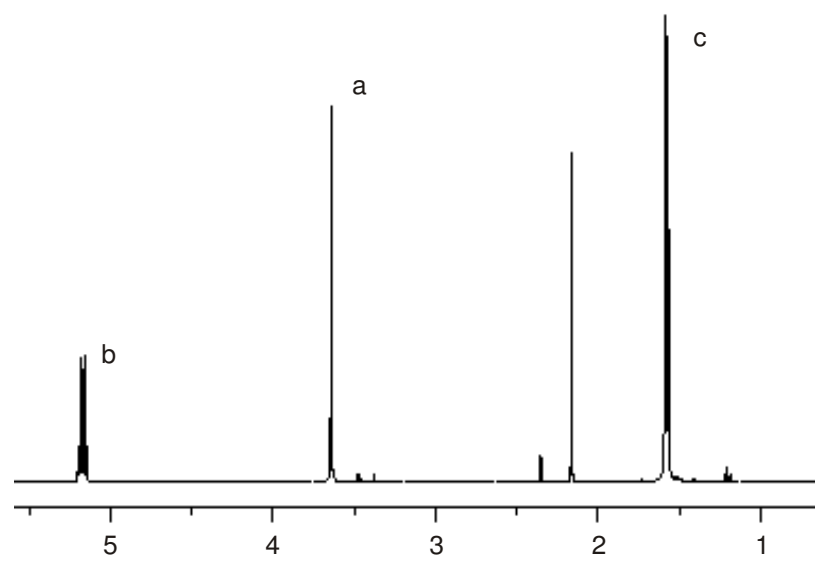

Fig. 1. ${ }^{1} \mathrm{H}$ NMR spectra $\left(400 \mathrm{MHz}, \mathrm{CDCl}_{3}\right)$ of $\mathrm{MPEG}_{1 \mathrm{~K}}-\mathrm{PLA}_{5 \mathrm{~K}}$

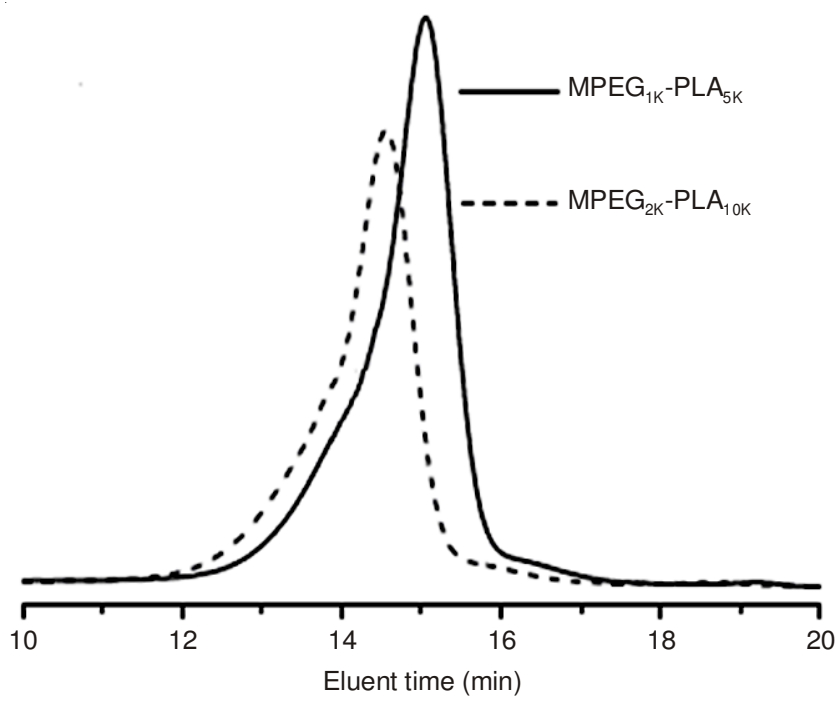

Fig. 2. GPC curves of $M P E G_{1 k}-P L A_{5 K}$ and $M P E G_{2 k}-P A_{10 K}$

Characterization of polymersomes: The basic properties of the copolymer and the polymersome with different ratios were shown in Table-1.

The polymersome sizes and morphologies were characterized by DLS and TEM. The spherical morphology of polymersome were well preserved as observed by TEM (Fig. 3) and the DLS results (Fig. 4). 


\begin{tabular}{|c|c|c|c|c|c|}
\hline \multicolumn{6}{|c|}{$\begin{array}{c}\text { TABLE-1 } \\
\text { PROPERTIES OF THE COPOLYMERS }\end{array}$} \\
\hline \multirow{2}{*}{ Sample number } & \multicolumn{2}{|c|}{ Molecular Ratio } & \multirow{2}{*}{ PDI } & \multirow{2}{*}{$\mathrm{Rh}(\mathrm{nm})$} & \multirow{2}{*}{$\mathrm{CO}-\mathrm{Hb}$ content $(\%)$} \\
\hline & Feed ratio & Product ratio & & & \\
\hline $\mathrm{mPEG}_{1 \mathrm{k}}-\mathrm{PLA}_{2 \mathrm{k}}$ & $1: 2$ & $1: 1.7$ & 1.54 & 17 & - \\
\hline $\mathrm{mPEG}_{1 \mathrm{k}}-\mathrm{PLA}_{5 \mathrm{k}}$ & $1: 5$ & 1:4.5 & 1.37 & 36 & - \\
\hline $\mathrm{mPEG}_{1 \mathrm{k}}-\mathrm{PLA}_{10 \mathrm{k}}$ & $1: 10$ & $1: 9$ & 1.62 & 45 & 28.9 \\
\hline $\mathrm{mPEG}_{2 \mathrm{k}}-\mathrm{PLA}_{10 \mathrm{k}}$ & $1: 5$ & $1: 4.7$ & 1.41 & 73 & 6.05 \\
\hline $\mathrm{mPEG}_{2 \mathrm{k}}-\mathrm{PLA}_{20 \mathrm{k}}$ & $1: 10$ & 1:9.6 & 1.35 & 64 & 13.65 \\
\hline $\mathrm{mPEG}_{2 \mathrm{k}}-\mathrm{PLA}_{30 \mathrm{k}}$ & $1: 15$ & $1: 14$ & 1.17 & 84 & 18.45 \\
\hline
\end{tabular}

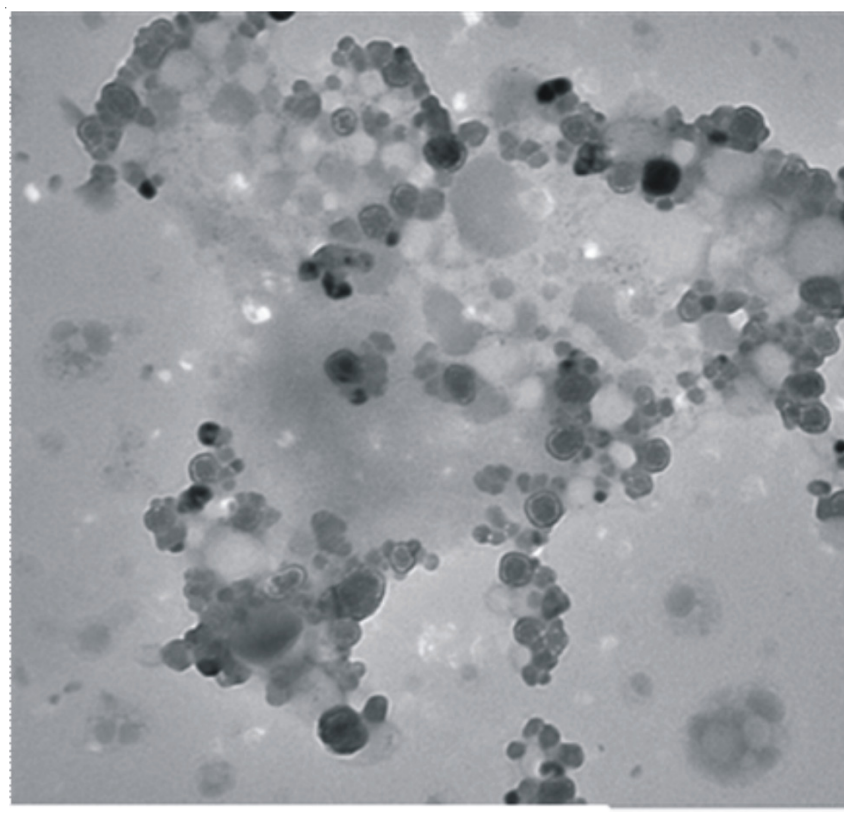

$500 \mathrm{~nm}$

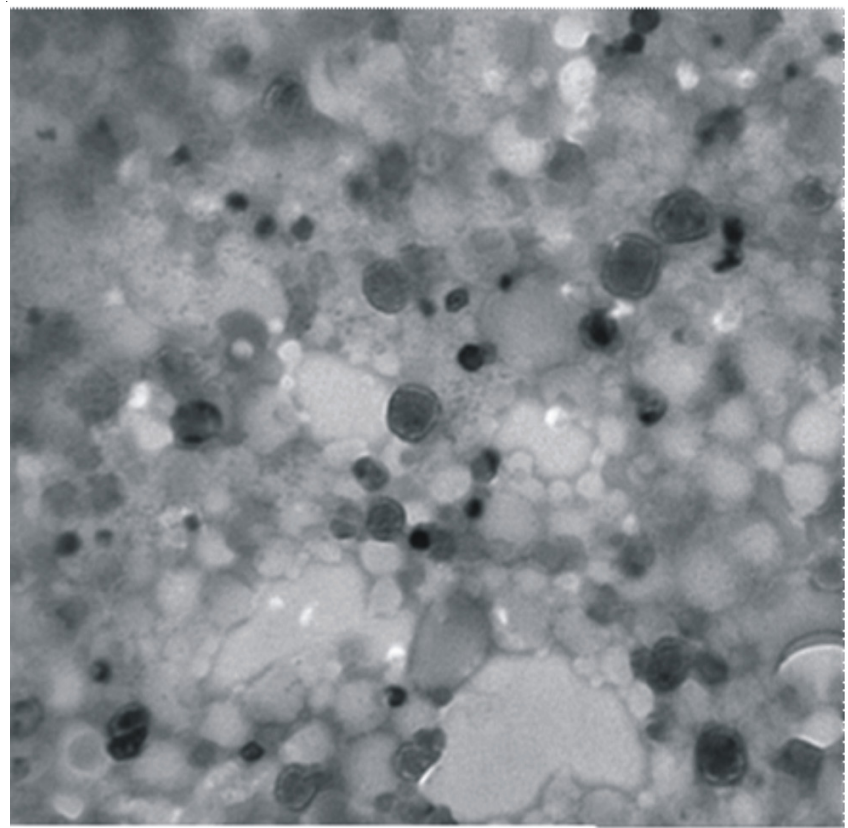

$500 \mathrm{~nm}$

Fig. 3. TEM images of Polymersomes prepared by $M P E G_{1 \mathrm{~K}}-\mathrm{PLA}_{10 \mathrm{~K}}$ (above) and $\mathrm{MPEG}_{2 \mathrm{~K}}-\mathrm{PLA}_{30 \mathrm{~K}}$ (below)

Characterization of hemoglobin-encapsulated polymersomes: The UV-visible spectra of CO-hemoglobin, oxyhemoglobin and deoxy-hemoglobin were described in Fig. 5.

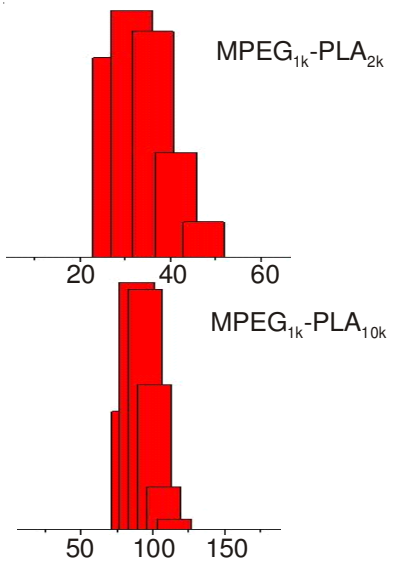

Fig. 4. DLS results of the polymersomes

The phenomenon that the absorption peaks were upward offset which because the polymersomes caused light scattering in the hemoglobin-encapsulated solution. UV visible spectra of the hemoglobin-encapsulated polymersomes under different oxygen partial pressures transformed from CO-hemoglobin to deoxy-hemoglobin were shown in Fig. 6. The polymersome was gradually transformed from CO-hemoglobin to deoxyhemoglobin by reducing the oxygen partial pressure. In conclusion that the hemoglobin encapsulated in the polymersome has independent binding capacity and deoxidation capacity, which means that the artificial blood nanoparticles has independent carrying oxygen function.

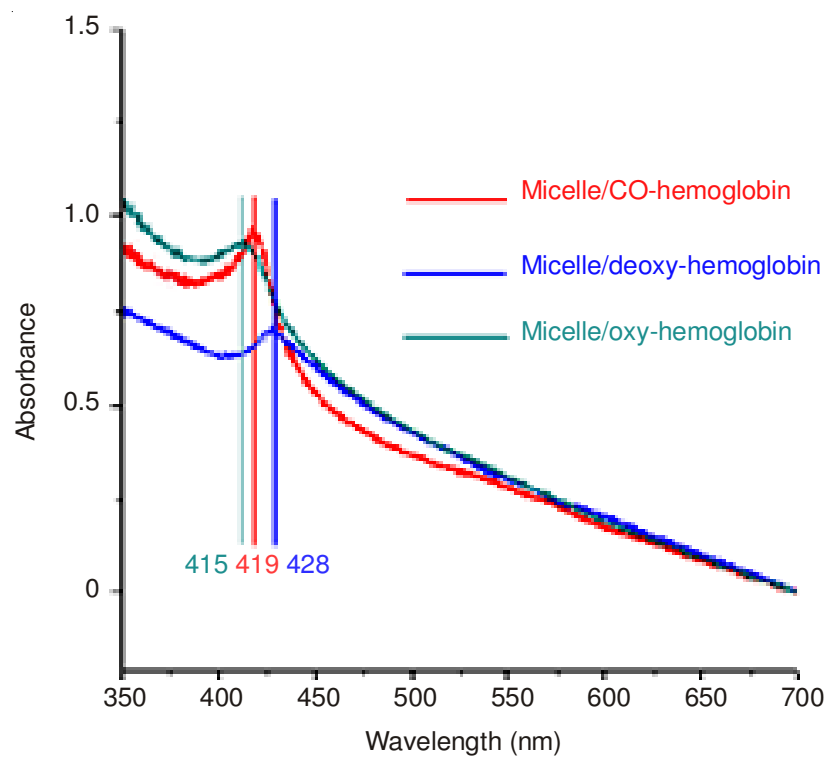

Fig. 5. UV spectra of the hemoglobin-encapsulated polymersome by MPEG $_{2 \mathrm{k}}-\mathrm{PLA}_{30 \mathrm{k}}$ 


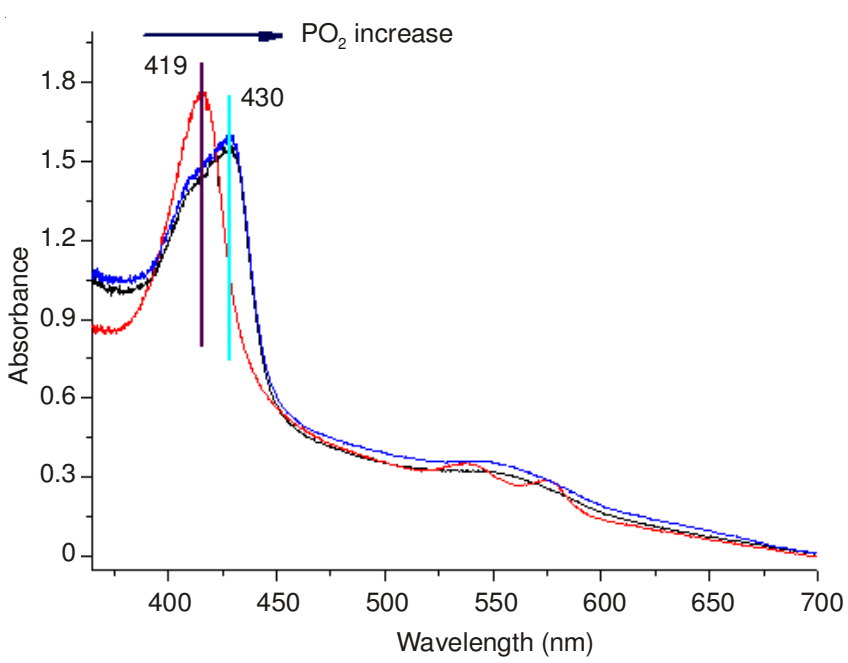

Fig. 6. UV spectra of hemoglobin-encapsulated polymersomes under different oxygen partial pressures transformed from CO-hemoglobin to deoxy-hemoglobin

\section{Conclusion}

The amphiphilic block copolymers MPEG-PLA were successfully synthetised and their structures were characterized by NMR. The molecular ratio and distribution were characterized by GPC. That was confirmed by dynamic light scattering and TEM. The optimization conditions were obtained that the hemoglobin was encapsulated in the polymersomes. The hemoglobin nanoparticles with different states encapsulated in the polymersomes can be mutual transformed under different gas atmosphere was observed by UV spectra. While the oxygen partial pressure was increased, the oxy-hemoglobin was gradually transformed from deoxy-hemoglobin. The hemoglobinencapsulated polymersomes can combined with the oxygen autonomously, which will be widely used in clinic to blood substitutes in future.

\section{REFERENCES}

1. H. Sakai, K. Sou, H. Horinouchi, K. Kobayashi and E. Tsuchida, Artif. Organs, 33, 139 (2009).

2. A.I. Alayash, Nat. Biotechnol., 17, 545 (1999).

3. L. Brander, A. Reil, J. Bux, B.M. Taleghani, B. Regli and J. Takala, Anesth. Analg., 101, 499 (2005).

4. A.W. Sellards and G.R. Minot, J. Med. Res., 34, 469 (1916).

5. W.R. Amberson, A.G. Mulder, F.R. Steggerda, J. Flexner and D.S. Pankratz, Science, 78, 106 (1933).

6. W.R. Amberson, J.J. Jennings and C.M. Rhode, J. Appl. Physiol., 1, 469 (1949).

7. B. Li, G. Chen, F.B. Meng, T.H. Li, J. Yue, X.B. Jing and Y.B. Huang, Polym. Chem., 3, 2421 (2012).

8. H. Agashe, P. Lagisetty, S. Awasthi and V. Awasthi, Colloids Surf. B, 75, 573 (2010).

9. B. Li, T.H. Li, G. Chen, X.Y. Li, L.S. Yan, Z.G. Xie, X.B. Jing and Y.B. Huang, Macromol. Biosci., 13, 893 (2013).

10. E.J. van Kampen and W.G. Zijlstra, Adv. Clin. Chem., 23, 199 (1983). 